DRAFT VERSION JULY 10, 2018

Preprint typeset using LTEX style emulateapj v. 03/07/07

\title{
Ultra-Luminous Supernovae as a New Probe of the Interstellar Medium in Distant Galaxies
}

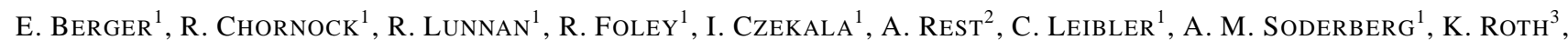
G. NArayan ${ }^{1}$, M. E. Huber ${ }^{4}$, D. Milisavljevic ${ }^{1}$, N. E. SAnders ${ }^{1}$, M. Drout ${ }^{1}$, R. Margutti ${ }^{1}$, R. P. Kirshner ${ }^{1}$, G. H. Marion ${ }^{1}$, P. J. Challis ${ }^{1}$, A. G. Riess ${ }^{2,5}$, S. J. SMartT ${ }^{6}$, W. S. BurgetT ${ }^{4}$, J. N. Heasley ${ }^{4}$, N. KAiser ${ }^{4}$, R.-P. Kudritzki ${ }^{4}$, E. A. MAgnier ${ }^{4}$, M. MCCrum ${ }^{6}$, P. A. Price ${ }^{7}$, K. SMith ${ }^{6}$, J. L. TOnRY ${ }^{4}$, And R. J. WAinsCoAT ${ }^{4}$

Draft version July 10, 2018

\begin{abstract}
We present the Pan-STARRS1 discovery and light curves, and follow-up MMT and Gemini spectroscopy of an ultra-luminous supernova (ULSN; dubbed PS1-11bam) at a redshift of $z=1.566$ with a peak brightness of $M_{\mathrm{UV}} \approx-22.3$ mag. PS1-11bam is one of the highest redshift spectroscopically-confirmed SNe known to date. The spectrum is characterized by broad absorption features typical of previous ULSNe (e.g., C II, Si III), and by strong and narrow Mg II and Fe II absorption lines from the interstellar medium (ISM) of the host galaxy, confirmed by an [O II] $\lambda 3727$ emission line at the same redshift. The equivalent widths of the Fe II $\lambda 2600$ and $\mathrm{Mg}$ II $\lambda 2803$ lines are in the top quartile of the quasar intervening absorption system distribution, but are weaker than those of gamma-ray burst intrinsic absorbers (i.e., GRB host galaxies). We also detect the host galaxy in pre-explosion Pan-STARRS1 data and find that its UV spectral energy distribution is best fit with a young stellar population age of $\tau_{*} \approx 15-45 \mathrm{Myr}$ and a stellar mass of $M_{*} \approx(1.1-2.6) \times 10^{9} \mathrm{M}_{\odot}$ (for $Z=0.05-1$ $\mathrm{Z}_{\odot}$ ). The star formation rate inferred from the UV continuum and [O II] $\lambda 3727$ emission line is $\approx 10 \mathrm{M}_{\odot} \mathrm{yr}^{-1}$, higher than in any previous ULSN host. PS1-11bam provides the first direct demonstration that ULSNe can serve as probes of the interstellar medium in distant galaxies. At the present, the depth and red sensitivity of PS1 are uniquely suited to finding such events at cosmologically interesting redshifts $(z \sim 1-2)$; the future combination of LSST and 30-m class telescopes promises to extend this technique to $z \sim 4$.
\end{abstract}

Subject headings: galaxies: ISM — supernovae: individual (PS1-11bam)— surveys: Pan-STARRS1

\section{INTRODUCTION}

Studies of the interstellar medium (ISM) in distant galaxies have traditionally focused on two observational techniques: (i) direct spectroscopic measurements of emission lines from the aggregate H II regions (e.g., Savaglio et al. 2005; Erb et al. 2006; Mannucci et al. 2009); and (ii) absorption by intervening systems in spectra of background quasars (e.g., Steidel \& Sargent 1992; Wolfe et al. 2005). These approaches are complementary: direct galaxy metallicity measurements at $z \gtrsim 2$ are challenging due to the relative faintness of galaxies and the redshifting of rest-frame optical emission lines into and beyond the near-IR band, while quasar absorption studies are most effective at $z \gtrsim 2$ where Ly $\alpha$ is redshifted into the observed optical window.

In recent years, these studies have been supplemented by absorption spectroscopy of bright gamma-ray burst (GRB) afterglows (e.g., Vreeswiik et al. 2004; Berger et al. 2006; Prochaska et al. 2007; Fynbo et al. 2009), which are detectable at least to $z \sim 9.5$ (Tanvir et al. 2009; Cucchiara et al. 2011). GRBs provide a unique view of the ISM since their massive star progenitors are embedded within star forming regions of their hosts (Bloom et al. 2002). As a result, GRBs

\footnotetext{
${ }^{1}$ Harvard-Smithsonian Center for Astrophysics, 60 Garden Street, Cambridge, MA 02138, USA

${ }^{2}$ Space Telescope Science Institute, 3700 San Martin Drive, Baltimore, Maryland 21218, USA

${ }^{3}$ Gemini Observatory, 670 N. Aohoku Place Hilo, HI 96720, USA

${ }^{4}$ Institute for Astronomy, University of Hawaii, 2680 Woodlawn Drive, Honolulu HI 96822

${ }^{5}$ Department of Physics and Astronomy, Johns Hopkins University, Baltimore, MD 21218, USA

${ }^{6}$ Astrophysics Research Centre, School of Mathematics and Physics, Queen's University Belfast, Belfast BT7 1NN, UK

${ }^{7}$ Department of Astrophysical Sciences, Princeton University, Princeton, NJ 08544, USA
}

probe galaxies (their hosts) at a small impact parameter of $\lesssim$ few kpc, and thus provide a complementary view to quasars, which tend to probe the outer halos of intervening galaxies due to the large cross-section at large radii. As a consequence of this critical difference, GRBs have revealed higher neutral hydrogen and metal column density absorbers than quasars show (e.g., Berger et al. 2006; Prochaska et al. 2007).

Despite this success, GRB observations are not trivial. First, they require wide-field $\gamma$-ray satellites with real-time arcminute-scale localization capability (e.g., Swift). Second, while GRB optical/near-IR afterglows are initially more luminous than quasars $(\langle m(1 \mathrm{hr})\rangle \sim 17 \mathrm{mag},\langle M(1 \mathrm{hr})\rangle \sim-28 \mathrm{mag}$; e.g., Kann et al. 2010), the emission fades rapidly, by 3-4 mag in one day. Thus, the window of opportunity for GRB absorption spectroscopy is brief. In this context, an alternative astrophysical source with the advantages of GRBs (embedded massive star progenitor, large luminosity), but that overcomes the disadvantages of GRBs (rapid fading, $\gamma$-ray discovery) would provide a powerful probe of distant galaxies. Normal supernovae ( $\mathrm{SNe}$ ) are inadequate for this purpose since with $M \gtrsim-19.5$ mag they are too faint for absorption spectroscopy at cosmologically interesting redshifts ${ }^{8}(z \gtrsim 1)$. Moreover, the spectral energy distributions (SEDs) of Type I SNe are strongly suppressed at $\lambda_{r} \lesssim 4000 \AA$ due to iron line blanketing, and they are therefore poorly suited to the detection of interstellar Ly $\alpha$ and metal UV lines in absorption. The SEDs of Type II SNe can extend into the UV, but they are generally less luminous than Type I events, although some Type IIn events are ultra-luminous (e.g., (Ofek et al. 2007; Smith et al. 2007)).

${ }^{8}$ Supernovae have been previously utilized for absorption studies of the ISM in nearby galaxies (e.g., Wallerstein et al. 1972; Vidal-Madjar et al. 1987; Bowen et al. 2000). 
Against this backdrop, a recently-discovered class of ultraluminous $\mathrm{SNe}$ (ULSNe) with $M \approx-22$ to $-23 \mathrm{mag}$ and SEDs that extend into the UV may provide a powerful probe of distant galaxies (Ouimby et al. 2007; Barbary et al. 2009; Pastorello et al. 2010; Chomiuk et al. 2011; Quimby et al. 2011). This potential was first noted by Ouimby et al. (2011), and further discussed by Chomiuk et al. (2011). Here we present the first demonstration of this potential with spectroscopic observations of an ULSN at $z=1.566$ discovered in the Pan-STARRS1 Medium-Deep Survey (PS1/MDS). This is one of the highest redshift spectroscopically-confirmed $\mathrm{SNe}$ known to date. The spectra exhibit interstellar absorption features from Fe II and Mg II with equivalent widths that are intermediate between the populations of quasar and GRB absorbers. We also present PS1 detections of the host galaxy in several rest-frame UV bands that point to a substantial star formation rate, a young stellar population, and low stellar mass that are reminiscent of GRB host galaxies. These results pave the way for the use of ULSNe as probes of distant galaxies in the LSST and GSMT era.

\section{OBSERVATIONS}

\subsection{PS1 Survey Summary}

The PS1 telescope on Haleakala is a high-etendue widefield survey instrument with a $1.8-\mathrm{m}$ diameter primary mirror and a $3.3^{\circ}$ diameter field of view imaged by an array of sixty $4800 \times 4800$ pixel detectors, with a pixel scale of $0.258^{\prime \prime}$ (Kaiser et al. 2010; Tonry \& Onaka 2009). The observations are obtained through five broad-band filters $\left(g_{\mathrm{P} 1} r_{\mathrm{P} 1} i_{\mathrm{P} 1} z_{\mathrm{P} 1} y_{\mathrm{P} 1}\right)$, with some differences relative to the Sloan Digital Sky Survey (SDSS); the $g_{\mathrm{P} 1}$ filter extends $200 \AA$ redward of $g_{\text {SDSS }}$ to achieve greater sensitivity and lower systematics for photometric redshifts, and the $z_{\mathrm{P} 1}$ filter terminates at $9300 \AA$, unlike $z_{\text {SDSS }}$ which is defined by the detector response (Tonry et al. 2012). PS1 photometry is in the "natural" system, $m=$ $-2.5 \log \left(F_{\nu}\right)+m^{\prime}$, with a single zero-point adjustment $\left(m^{\prime}\right)$ in each band to conform to the AB magnitude scale. Magnitudes are interpreted as being at the top of the atmosphere, with 1.2 airmasses of atmospheric attenuation included in the system response function (Tonry et al. 2012).

The PS1 Medium-Deep Survey (MDS) consists of 10 fields (each with a single PS1 imager footprint) observed on a nearly nightly basis by cycling through the five filters in 3-4 nights to a $5 \sigma$ depth of $\sim 23.3 \mathrm{mag}$ in $g_{\mathrm{P} 1} r_{\mathrm{P} 1} i_{\mathrm{P} 1} z_{\mathrm{P} 1}$, and $\sim 21.7 \mathrm{mag}$ in $y_{\mathrm{P} 1}$. The MDS images are processed through the Image Processing Pipeline (IPP; Magnier 2006), which includes flatfielding ("de-trending"), a flux-conserving warping to a skybased image plane, masking and artifact removal, and object detection and photometry; transient detection using IPP photometry is carried out at Queen's University Belfast. Independently, difference images are produced from the stacked nightly images by the photpipe pipeline (Rest et al. 2005) running on the Odyssey computer cluster at Harvard University. The discovery and data presented here are from the photpipe analysis.

\subsection{Discovery and Photometric Observations of PS1-11bam}

PS1-11bam was discovered in PS1/MDS data at $\mathrm{RA}=08^{\mathrm{h}} 41^{\mathrm{m}} 14.192^{\mathrm{s}}$, Dec $=+44^{\circ} 01^{\prime} 56.95^{\prime \prime}(\mathrm{J} 2000)$, about 10 days before maximum light, with the time of peak corresponding to 2011 November 22 UT (Figure 1). The object was detected in the first images of the season, so the actual rise time is likely $\gtrsim 10 \mathrm{~d}$. PS1-11bam was detected for nearly
$100 \mathrm{~d}$ in the $i_{\mathrm{P} 1}$ and $z_{\mathrm{P} 1}$ bands, and for a shorter period of time near peak in the $g_{\mathrm{P} 1}$ and $r_{\mathrm{P} 1}$ bands. The peak absolute magnitude in $i_{\mathrm{P} 1}\left(\lambda_{r} \approx 2930 \AA\right)$ is $M_{\mathrm{AB}}=-22.3 \pm 0.1 \mathrm{mag}$, matching the most luminous $\mathrm{SNe}$ to date (c.f., Chomiuk et al. 2011; Quimby et al. 2011).

The SED within $\pm 1 \mathrm{~d}$ of peak brightness can be fit with a blackbody function, with a temperature of about $1.7 \times 10^{4} \mathrm{~K}$ (Figure 11). The flux density in the $g_{\mathrm{P} 1}$-band $\left(\lambda_{r} \approx 1880 \AA\right)$ is suppressed compared to the blackbody model due to broad absorption blueward of $\lambda_{r} \approx 2000 \AA$ ( $\$ 2.3$ and Figure 1 ).

\subsection{Spectroscopy of PS1-11bam}

We obtained spectra of PS1-11bam with the Blue Channel spectrograph (Schmidt et al. 1989) on the MMT 6.5-m telescope on 2011 November 29 UT. The spectrum consisted of $3 \times 1200 \mathrm{~s}$ exposures obtained at an airmass of 1.1 with a $1^{\prime \prime}$ slit aligned at the parallactic angle. The data were processed using standard procedures in IRAF, and the resulting spectrum covers $\approx 3320-8530 \AA$. As shown in Figure 1 , the spectrum exhibits broad absorption features at about 4500 , 5650, 6150, and $6800 \AA$, corresponding to features seen in previous ULSNe (Quimby et al. 2007; Pastorello et al. 2010; Chomiuk et al. 2011; Quimby et al. 2011). Accounting for an expansion velocity of about $-15,000 \mathrm{~km} \mathrm{~s}^{-1}$ (Chomiuk et al. 2011), the resulting redshift is $z \approx 1.55$. At this redshift, emission is detected to $\lambda_{r} \approx 1300 \AA$.

Following this identification we obtained additional spectra with the Gemini Multi-Object Spectrograph (GMOS; Hook et al. 2004) on the Gemini North 8-m telescope on 2011 December 5 and 2012 January 1 UT. The observations consisted of $2 \times 1500 \mathrm{~s}$ exposures with the R400 grating (December 5), $2 \times 1200 \mathrm{~s}$ with the B600 grating (January 1), and $2 \times 1050 \mathrm{~s}$ with the R400 grating (January 1) all taken at airmass of $1.1-1.2$ and with a $1^{\prime \prime}$ slit aligned at the parallactic angle. The spectra were processed using the gemini package in IRAF, and cover 4800-9050 $\AA$ and 3850-9600 $\AA$, respectively, with a resolution of about $7 \AA$. In addition to the broad SN features, the first Gemini spectrum reveals narrow absorption lines of Fe II and $\mathrm{Mg}$ II at a common redshift of $z=1.5657 \pm 0.0003$ (Table 1 and Figure 2). The second Gemini spectrum further reveals a narrow [O II] $\lambda 3727$ emission line at $z=1.567 \pm 0.001$ (Figure 2), confirming that the absorption features arise in the host galaxy of PS1-11bam, with a potential velocity offset of $-150 \pm 80 \mathrm{~km} \mathrm{~s}^{-1}$. The emission/absorption redshift also validates our identification of the broad SN features.

The detailed properties of PS1-11bam will be discussed in a separate paper (Lunnan et al. in prep.); here we focus on the interstellar absorption and the properties of the associated host galaxy.

\section{INTERSTELLAR ABSORPTION AND COMPARISON TO QUASARS AND GAMMA-RAY BURST ABSORBERS}

The detected interstellar Fe II and Mg II features are shown in Figure 2 and summarized in Table 11. The rest-frame equivalent widths of the strongest lines are $W_{r}(\mathrm{Fe}$ II $\lambda 2600)=$ $1.2 \pm 0.1 \AA$ and $W_{r}(\operatorname{Mg}$ II $\lambda 2803)=1.3 \pm 0.2 \AA$. We use the Mg II $\lambda 2803$ line since the Mg II $\lambda 2796$ line is mildly contaminated by an atmospheric sky line. The equivalent widths allow us to place a lower limit on the ion column densities, assuming the optically thin regime of the curve-of-growth, $N \gtrsim 1.13 \times 10^{20} \mathrm{~cm}^{-2}\left(W_{r} / f \lambda_{r}^{2}\right)$; here $W_{r}$ and $\lambda_{r}$ are rest-frame values in units of $\AA$ and $f$ is the oscillator strength. This is 
a lower limit due to likely line saturation and the low spectral resolution. From the strong lines we find $\log N(\mathrm{Fe}$ II $) \gtrsim$ 13.9 and $\log N(\mathrm{Mg}$ II $) \gtrsim 13.8$, although the weakest oscillator strength line (Fe II $\lambda 2374$ ) gives $\log N(\mathrm{Fe}$ II) $\gtrsim 14.7$. We note that the redshift of PS1-11bam is not large enough for a detection of $\operatorname{Ly} \alpha$, and we therefore cannot determine the gas-phase metallicity.

In Figure 3 we compare the Fe II $\lambda 2600$ and $\mathrm{Mg}$ II $\lambda 2803$ equivalent widths to those measured for quasar intervening systems at $z \approx 0.4-2.3$ from the Sloan Digital Sky Survey (Quider et al. 2011), and for intrinsic absorbers (i.e., host galaxies) from GRB spectra (Fynbo et al. 2009). We find that the equivalent widths for PS1-11bam are in the top quartile of the quasar absorption system distribution, but are in the bottom quartile of the GRB intrinsic absorbers. In comparison to the composite GRB spectrum of Christensen et al. (2011), the $\mathrm{Mg}$ II $\lambda 2803$ equivalent width is comparable, with $1.3 \AA$ for PS1-11bam and $1.5 \AA$ for the GRB composite. The Fe II $\lambda 2600$ equivalent width is lower, with $1.2 \AA$ for PS111 bam and $1.85 \AA$ for the GRB composite. However, we note that the Fe II $\lambda 2600$ line in the GRB composite spectrum is blended with the fine-structure line Fe II* $\lambda 2599$, so the actual equivalent width is $\lesssim 1.85 \AA$. The fine-structure line is due to UV pumping by the early afterglow intense radiation field (Dessauges-Zavadsky et al. 2006; Vreeswijk et al. 2007), which we do not expect ${ }^{9}$ in the case of PS1-11bam. Finally, we find that the equivalent widths for PS1-11bam are about 3 times smaller than the values measured from a composite spectrum of 13 star forming galaxies at a mean redshift of $z \approx 1.6$ from the Gemini Deep Deep Survey (GDDS; Savaglio et al. 2004). For subsequent comparison with the host of PS1-bam (\$4), we note that the GDDS galaxies have a mean rest-frame UV absolute magnitude of $M_{2000} \approx-20.3$ $\mathrm{AB}$ mag.

The larger equivalent widths for the PS1-11bam absorber compared to the bulk of quasar intervening systems, and the similarity to the GRB composite spectrum, which is mainly composed of damped Ly $\alpha$ absorbers (DLAs; $\log N(\mathrm{HI}) \gtrsim$ 20.3), suggests that PS1-11bam would also uncover a DLA if we could measure its $\operatorname{Ly} \alpha$ column density. Indeed, quasar studies suggest that about $80 \%$ of all absorbers with $\operatorname{Mg}$ II $\lambda 2796 \gtrsim 1.3 \AA$, and about $50 \%$ of absorbers with Fe II $\lambda 2600 \gtrsim 1.2 \AA$ are DLAs (Rao et al. 2006).

\section{HOST GALAXY PROPERTIES}

We detect the host galaxy of PS1-11bam in pre-explosion stacks of the PS1/MDS data with apparent magnitudes ${ }^{10}$ of $g_{\mathrm{P} 1}=23.62 \pm 0.13, r_{\mathrm{P} 1}=23.62 \pm 0.12, i_{\mathrm{P} 1}=23.78 \pm 0.13$, $z_{\mathrm{P} 1}=23.73 \pm 0.14$, and $y_{\mathrm{P} 1} \gtrsim 23.4(3 \sigma)$; see Figure 4. The blue colors of the galaxy are indicative of a young stellar population. The host SED covers rest-frame wavelengths of about $1900-3800 \AA$, with the $y_{\mathrm{P} 1}$-band limit constraining the $4000 \AA /$ Balmer break (Figure 4). We fit the SED with the Maraston (2005) evolutionary stellar population synthesis models, using a Salpeter initial mass function and a red horizontal branch morphology, with the stellar population age $\left(\tau_{*}\right)$ and stellar mass $\left(M_{*}\right)$ as free parameters. For a metallicity range of $0.05-1 \mathrm{Z}_{\odot}$ we find $\tau_{*} \approx 15-45 \mathrm{Myr}$ and $M_{*} \approx(1.1-2.6) \times 10^{9} \mathrm{M}_{\odot}\left(\chi^{2}=1.2\right.$ for 3 degrees of freedom;

\footnotetext{
${ }^{9}$ Fine-structure lines may be excited in the local circumstellar medium in the case of strongly-interacting SNe (e.g., Bowen et al. 2000).

10 These values are corrected for Galactic extinction of $E(B-V) \approx 0.026$ mag (Schlegel et al. 1998).
}

Figure 4); lower metallicity leads to older ages and larger stellar masses. We also find that $A_{V}^{\text {host }} \lesssim 0.5$ mag for an assumed LMC extinction curve.

From the observed $g_{\mathrm{P} 1}$-band we infer a rest-frame UV luminosity of $L_{\nu, \mathrm{UV}} \approx 8 \times 10^{28} \mathrm{erg} \mathrm{s}^{-1} \mathrm{~Hz}^{-1}$, corresponding to a star formation rate of SFR $\approx 12 \mathrm{M}_{\odot} \mathrm{yr}^{-1}$ (Kennicutt 1998). The upper bound on the extinction $\left(A_{1900}^{\text {host }} \approx 1.3 \mathrm{mag}\right)$ indicates that $\mathrm{SFR} \lesssim 40 \mathrm{M}_{\odot} \mathrm{yr}^{-1}$. From the [O II] $\lambda 3727$ line flux, $F([\mathrm{OII}]) \approx 7 \times 10^{-17} \mathrm{erg} \mathrm{cm}^{-2} \mathrm{~s}^{-1}$, we find SFR $\approx 6$ $\mathrm{M}_{\odot} \mathrm{yr}^{-1}$ (Kennicutt 1998). The star formation rate is substantially larger than for previous ULSNe hosts, with SFR $\approx$ $0.1-1 \mathrm{M}_{\odot} \mathrm{yr}^{-1}$ (Chomiuk et al. 2011; Neill et al. 2011). The young stellar population age, low stellar mass, and appreciable star formation rate are reminiscent of long GRB host galaxies, for which $\left\langle\tau_{*}\right\rangle \sim 60 \mathrm{Myr},\left\langle M_{*}\right\rangle \sim 1.5 \times 10^{9} \mathrm{M}_{\odot}$, and $\langle\mathrm{SFR}\rangle \sim 10 \mathrm{M}_{\odot} \mathrm{yr}^{-1}$ (Christensen et al. 2004; Savaglio et al. 2009; Leibler \& Berger 2010).

The rest-frame UV absolute magnitude of the host galaxy of PS1-11bam, $M_{2000} \approx-20.7 \mathrm{AB}$ mag, is brighter than the mean of the GDDS composite spectrum, $M_{2000} \approx-20.3 \mathrm{AB}$ mag (Savaglio et al. 2004). This may appear puzzling given the much larger Fe II $\lambda 2600$ and $\mathrm{Mg}$ II $\lambda 2803$ equivalent widths in the GDDS composite compared to PS1-11bam, but we note that the GDDS galaxies are $K$-band (i.e., stellar mass) selected, while the host of PS1-11bam was selected through a massive star explosion (i.e., star formation). As a result, despite its larger UV luminosity, the host of PS1-11bam is most likely a lower stellar mass system than the mean GDDS galaxy.

\section{DISCUSSION AND CONCLUSIONS}

We presented the discovery of the ULSN PS1-11bam, and follow-up spectroscopy that reveals broad SN features and interstellar absorption from Fe II and Mg II. The ISM line equivalent widths are larger than the median of the quasar intervening absorber sample, but smaller than the GRB intrinsic absorber sample. They are substantially lower compared to a stack of GDDS galaxies at $z \approx 1.6$. Although the potential of ULSNe as probes of distant galaxies has been proposed previously (Quimby et al. 2011), this is the first direct demonstration of this approach at a cosmologically interesting redshift that overlaps with the GRB and quasar samples. This is also the redshift range at which direct galaxy metallicity measurements become progressively more challenging.

We also detect the host galaxy of PS1-11bam in preexplosion images, and find that it exhibits a substantial star formation rate, a young stellar population age, and a low stellar mass, similar to the long GRB host sample. The star formation rate is substantially larger than in previous ULSN hosts (Chomiuk et al. 2011; Neill et al. 2011), indicating that they likely span a wide range of properties.

The spectrum of PS1-11bam demonstrates that ISM measurements using ULSNe are both promising and challenging. First, the luminosities are about a factor of 10-100 times lower than for GRB afterglows, but this can be partially overcome with repeated observations during the broad peak, and in the future with 30-m class telescopes. Second, with a typical blackbody temperature of $\sim 2 \times 10^{4} \mathrm{~K}$, the continuum flux at $\lambda_{r} \sim 1200 \AA$, required for absorption measurements of Ly $\alpha$, is about 5 times lower than at the peak of the SED. However, our observations of PS1-11bam demonstrate that continuum emission is indeed detectable to at least $\lambda_{r} \sim 1300 \AA$. Third, any broad SN features near $\lambda_{r} \sim 1200 \AA$ may com- 
plicate measurements of $\operatorname{Ly} \alpha$ absorption; existing data do not extend sufficiently blueward to assess this point. Fourth, since circumstellar (CSM) interaction has been invoked to explain the large luminosities of ULSNe (Chevalier \& Irwin 2011), absorption in the CSM may complicate measurements of ISM features. However, CSM interaction is generally accompanied by strong emission or P Cygni line profiles, which are also time variable (e.g., Bowen et al.2000). This is not seen in PS1-11bam, and it may serve as a discriminant between ISM and CSM absorption for future events. Finally, our observations demonstrate that at present PS1 is uniquely capable of discovering UV-bright ULSNe to $z \sim 2$, thanks to its superior red sensitivity and use of $i_{\mathrm{P} 1} z_{\mathrm{P} 1}$-band filters, and that followup spectroscopy with 8-m class telescopes can probe ISM absorption features; the upcoming Dark Energy Survey and PS2 will have the same potential. With the even greater depth of LSST, in conjunction with follow-up spectroscopy from 30$\mathrm{m}$ class telescopes, ULSNe will ultimately probe galaxies to $z \sim 4$.

PS1 has been made possible through contributions of the Institute for Astronomy, the University of Hawaii, the PanSTARRS1 Project Office, the Max-Planck Society and its participating institutes, the Max Planck Institute for Astronomy, Heidelberg and the Max Planck Institute for Extrater- restrial Physics, Garching, The Johns Hopkins University, Durham University, the University of Edinburgh, Queen's University Belfast, the Harvard-Smithsonian Center for Astrophysics, and the Las Cumbres Observatory Global Telescope Network, Incorporated, the National Central University of Taiwan, and the National Aeronautics and Space Administration under Grant NNX08AR22G issued through the Planetary Science Division of the NASA Science Mission Directorate. This work is based in part on observations obtained at the Gemini Observatory (Program GN-2011B-Q-3; PI: Berger), which is operated by the Association of Universities for Research in Astronomy, Inc., under a cooperative agreement with the NSF on behalf of the Gemini partnership: the National Science Foundation (United States), the Science and Technology Facilities Council (United Kingdom), the National Research Council (Canada), CONICYT (Chile), the Australian Research Council (Australia), Ministério da Ciência, Tecnologia e Inovação (Brazil) and Ministerio de Ciencia, Tecnología e Innovación Productiva (Argentina). Observations were also obtained at the MMT Observatory, a joint facility of the Smithsonian Institution and the University of Arizona. Some of the computations in this paper were run on the Odyssey cluster supported by the FAS Science Division Research Computing Group at Harvard University.

\section{REFERENCES}

Barbary, K., et al. 2009, ApJ, 690, 1358

Berger, E., Penprase, B. E., Cenko, S. B., Kulkarni, S. R., Fox, D. B., Steidel, C. C., \& Reddy, N. A. 2006, ApJ, 642, 979

Bloom, J. S., Kulkarni, S. R., \& Djorgovski, S. G. 2002, AJ, 123, 111

Bowen, D. V., Roth, K. C., Meyer, D. M., \& Blades, J. C. 2000, ApJ, 536, 225

Chevalier, R. A., \& Irwin, C. M. 2011, ApJ, 729, L6

Chomiuk, L., et al. 2011, ApJ, 743, 114

Christensen, L., Fynbo, J. P. U., Prochaska, J. X., Thöne, C. C., de Ugarte Postigo, A., \& Jakobsson, P. 2011, ApJ, 727, 73

Christensen, L., Hjorth, J., \& Gorosabel, J. 2004, A\&A, 425, 913

Cucchiara, A., et al. 2011, ApJ, 736, 7

Dessauges-Zavadsky, M., Chen, H.-W., Prochaska, J. X., Bloom, J. S., \& Barth, A. J. 2006, ApJ, 648, L89

Erb, D. K., Shapley, A. E., Pettini, M., Steidel, C. C., Reddy, N. A., \& Adelberger, K. L. 2006, ApJ, 644, 813

Fynbo, J. P. U., et al. 2009, ApJS, 185, 526

Hook, I. M., Jørgensen, I., Allington-Smith, J. R., Davies, R. L., Metcalfe, N., Murowinski, R. G., \& Crampton, D. 2004, PASP, 116, 425

Kaiser, N., et al. 2010, in Society of Photo-Optical Instrumentation Engineers (SPIE) Conference Series, Vol. 7733, Society of Photo-Optical Instrumentation Engineers (SPIE) Conference Series

Kann, D. A., et al. 2010, ApJ, 720, 1513

Kennicutt, R. C., Jr. 1998, ARA\&A, 36, 189

Leibler, C. N., \& Berger, E. 2010, ApJ, 725, 1202

Magnier, E. 2006, in The Advanced Maui Optical and Space Surveillance Technologies Conference

Mannucci, F., et al. 2009, MNRAS, 398, 1915

Maraston, C. 2005, MNRAS, 362, 799

Neill, J. D., et al. 2011, ApJ, 727, 15

Ofek, E. O., et al. 2007, ApJ, 659, L13

Pastorello, A., et al. 2010, ApJ, 724, L16
Prochaska, J. X., Chen, H.-W., Dessauges-Zavadsky, M., \& Bloom, J. S. 2007, ApJ, 666, 267

Prochaska, J. X., Gawiser, E., Wolfe, A. M., Cooke, J., \& Gelino, D. 2003, ApJS, 147, 227

Quider, A. M., Nestor, D. B., Turnshek, D. A., Rao, S. M., Monier, E. M., Weyant, A. N., \& Busche, J. R. 2011, AJ, 141, 137

Quimby, R. M., Aldering, G., Wheeler, J. C., Höflich, P., Akerlof, C. W., \& Rykoff, E. S. 2007, ApJ, 668, L99

Quimby, R. M., et al. 2011, Nature, 474, 487

Rao, S. M., Turnshek, D. A., \& Nestor, D. B. 2006, ApJ, 636, 610

Rest, A., et al. 2005, ApJ, 634, 1103

Savaglio, S., et al. 2004, ApJ, 602, 51

Savaglio, S., Glazebrook, K., \& Le Borgne, D. 2009, ApJ, 691, 182

Savaglio, S., et al. 2005, ApJ, 635, 260

Schlegel, D. J., Finkbeiner, D. P., \& Davis, M. 1998, ApJ, 500, 525

Schmidt, G. D., Weymann, R. J., \& Foltz, C. B. 1989, PASP, 101, 713

Smith, N., et al. 2007, ApJ, 666, 1116

Steidel, C. C., \& Sargent, W. L. W. 1992, ApJS, 80, 1

Tanvir, N. R., et al. 2009, Nature, 461, 1254

Tonry, J., \& Onaka, P. 2009, in Advanced Maui Optical and Space Surveillance Technologies Conference,

Tonry, J. L., et al. 2012, ApJ, 750, 99

Vidal-Madjar, A., Andreani, P., Cristiani, S., Ferlet, R., Lanz, T., \& Vladilo, G. 1987, A\&A, 177, L17

Vreeswijk, P. M., et al. 2004, A\&A, 419, 927

Vreeswijk, P. M., et al. 2007, A\&A, 468, 83

Wallerstein, G., Conti, P. S., \& Greenstein, J. L. 1972, Astrophys. Lett., 12, 101

Wolfe, A. M., Gawiser, E., \& Prochaska, J. X. 2005, ARA\&A, 43, 861 
TABLE 1

ISM ABSORPTION LINES IN PS1-11BAM

\begin{tabular}{cccccc}
\hline \hline $\begin{array}{c}\lambda_{\text {obs }} \\
(\AA)\end{array}$ & $\begin{array}{c}\text { Line } \\
(\AA)\end{array}$ & $f_{i j}$ & $z$ & $\begin{array}{c}W_{r} \\
(\AA)\end{array}$ & $\begin{array}{c}\log N \\
\left(\mathrm{~cm}^{-2}\right)\end{array}$ \\
\hline 6014.29 & Fe II 2344.214 & 0.114 & 1.5656 & $1.0 \pm 0.2$ & 14.3 \\
6092.81 & Fe II 2374.461 & 0.031 & 1.5660 & $0.7 \pm 0.2$ & 14.7 \\
6114.45 & Fe II 2382.765 & 0.320 & 1.5661 & $0.7 \pm 0.2$ & 13.6 \\
6638.80 & Fe II 2586.650 & 0.069 & 1.5666 & $0.8 \pm 0.2$ & 14.3 \\
6672.35 & Fe II 2600.173 & 0.239 & 1.5661 & $1.2 \pm 0.1$ & 13.9 \\
7174.50 & Mg II 2796.352 & 0.612 & 1.5657 & $1.0 \pm 0.2$ & 13.4 \\
7192.93 & Mg II 2803.531 & 0.305 & 1.5657 & $1.3 \pm 0.2$ & 13.8 \\
\hline
\end{tabular}



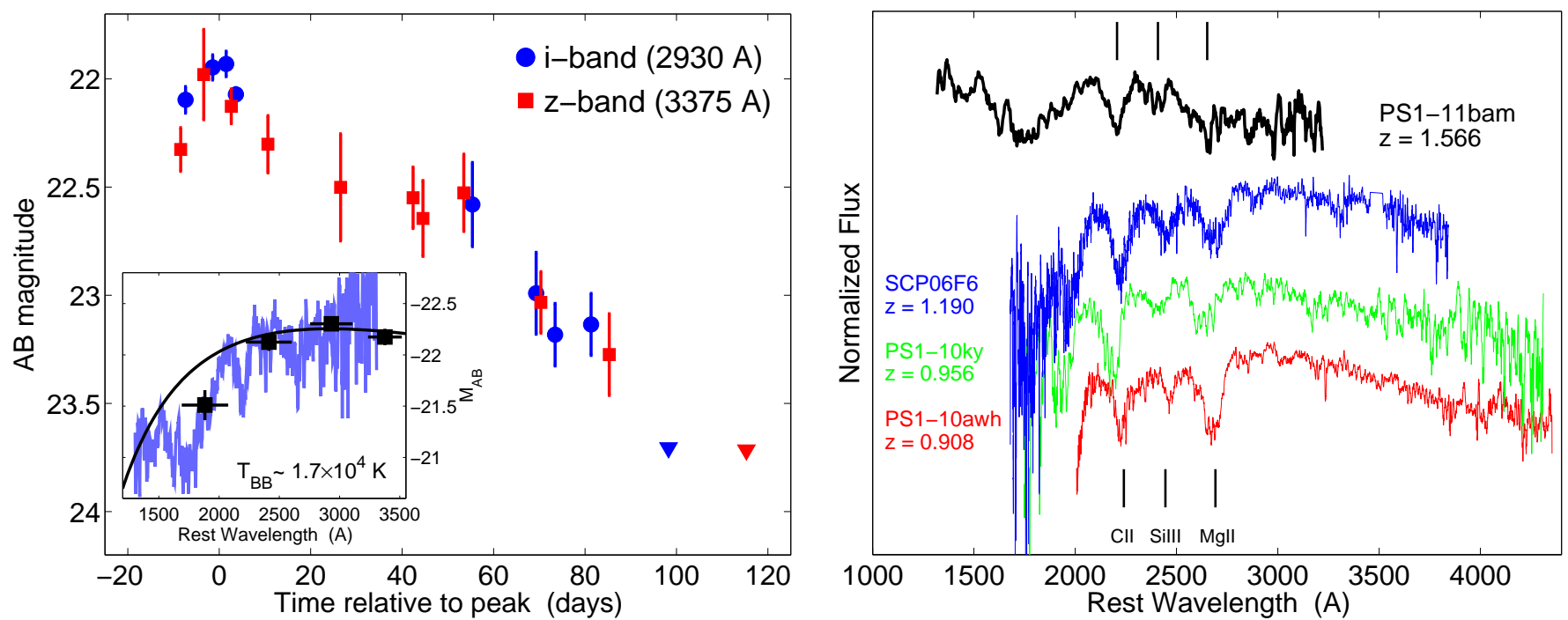

FIG. 1.- Left: PS1 $i_{\mathrm{P} 1 z_{\mathrm{P} 1}}$ light curves of PS1-11bam in the observer frame. We define the peak time relative to the $i_{\mathrm{P} 1}$ data. The transient was discovered about $10 \mathrm{~d}$ before maximum in the first images of the year and faded below the PS1/MDS detection threshold at about $80 \mathrm{~d}$ post-maximum. The inset shows the SED from $g_{\mathrm{P} 1} r_{\mathrm{P} 1} i_{\mathrm{P} 1} z_{\mathrm{P} 1}$ data obtained within $\pm 1 \mathrm{~d}$ of the peak, as well as the MMT spectrum scaled by the $i_{\mathrm{P} 1}$-band magnitude. The SED matches a blackbody spectrum with $T_{\mathrm{BB}} \approx 1.7 \times 10^{4} \mathrm{~K}$, with a suppression in $g_{\mathrm{P} 1}$ due to broad absorption at $\lambda_{r} \lesssim 2000 \AA$ A. Right: MMT spectrum of PS1-11bam (black) exhibiting broad absorption features typical of previous ULSNe (C II, Si III, and Mg II). For comparison we show the three previous highest-redshift ULSNe: SCP06F6 at $z=1.190$ (blue; Barbary et al. 2009), PS1-10ky at $z=0.956$ (green; Chomiuk et al. 2011), and PS1-10awh at $z=0.908$ (red; Chomiuk et al. 2011). Flux from PS1-11bam is detected to at least $\lambda_{r} \approx 1300 \AA$. 

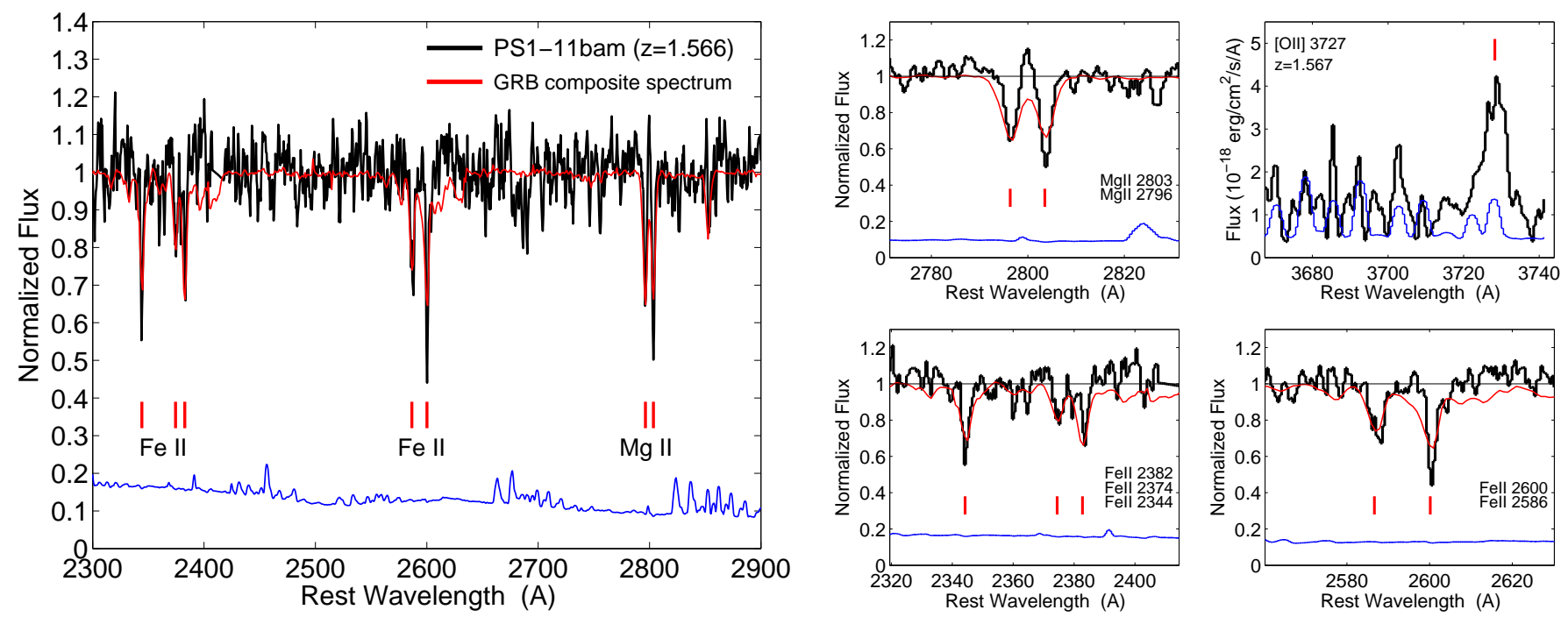

FIG. 2. - Left: Portion of the Gemini spectrum of PS1-11bam from December 5 containing several interstellar absorption features of Fe II and Mg II at $z=1.566$ (black). The error spectrum is shown in blue. For comparison we plot the GRB composite spectrum of Christensen et al. (2011). Right: A zoom-in on the relevant Fe II and Mg II lines demonstrates the similarity to GRB absorption spectra. Also shown is the [O II] $] 3727$ emission line at $z=1.567$ from the January 1 Gemini spectrum. 


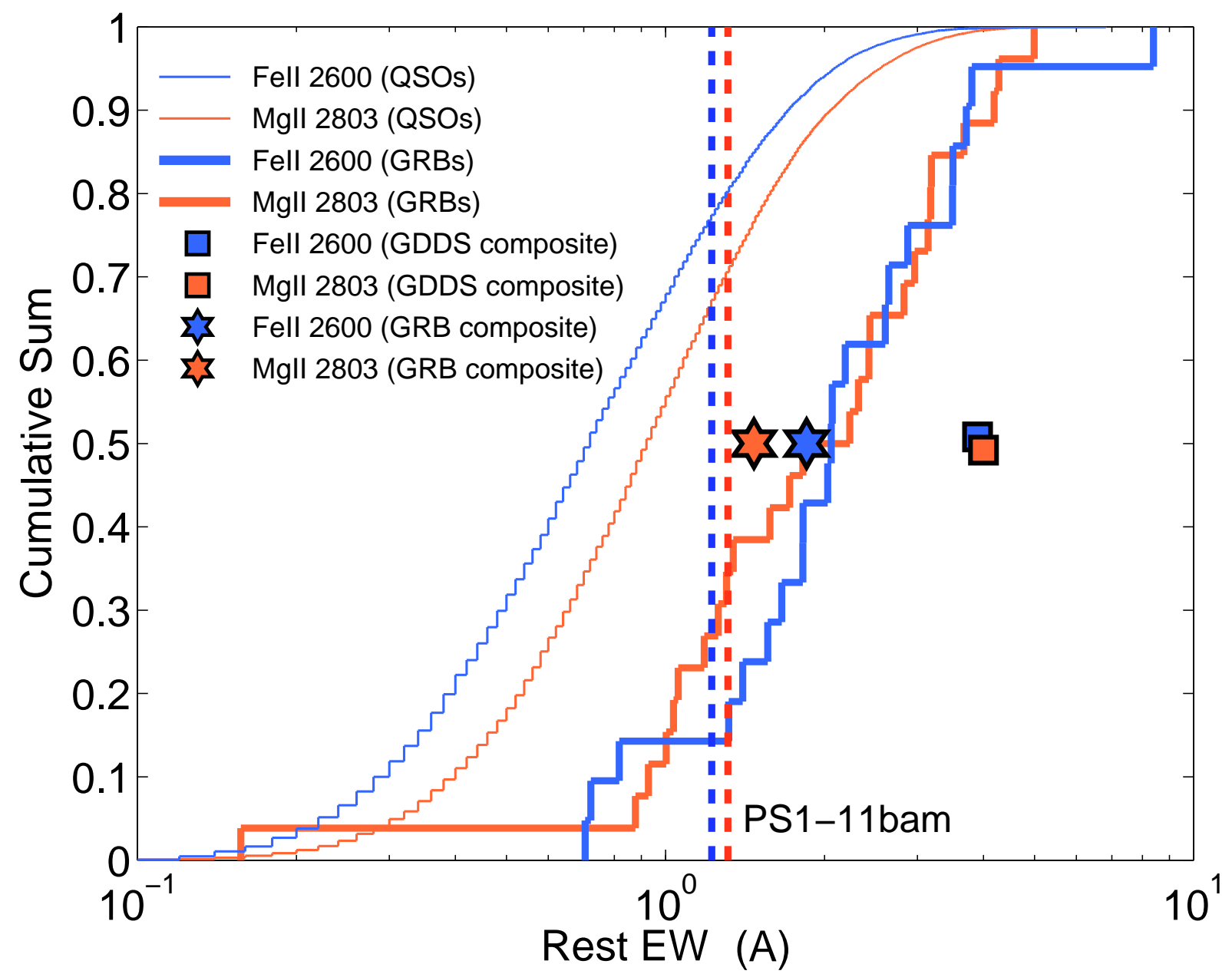

FIG. 3. - Rest-frame equivalent widths of Mg II $\lambda 2803$ (orange dashed vertical line) and Fe II $\lambda 2600$ (blue dashed vertical line) for PS1-11bam. Also shown are the equivalent width distributions for intervening systems at $z \approx 0.4-2.3$ from SDSS quasar absorption spectra (thin lines; Quider et al. 2011), intrinsic absorbers from GRB spectra (thick lines; Fynbo et al. 2009), the values from a GRB composite spectrum (hexagrams; Christensen et al. (2011)), and the values from a stack of 13 star forming galaxies at $z \approx 1.3-2$ from the Gemini Deep Deep Survey (squares, offset vertically for clarity; Savaglio et al. 2004). 

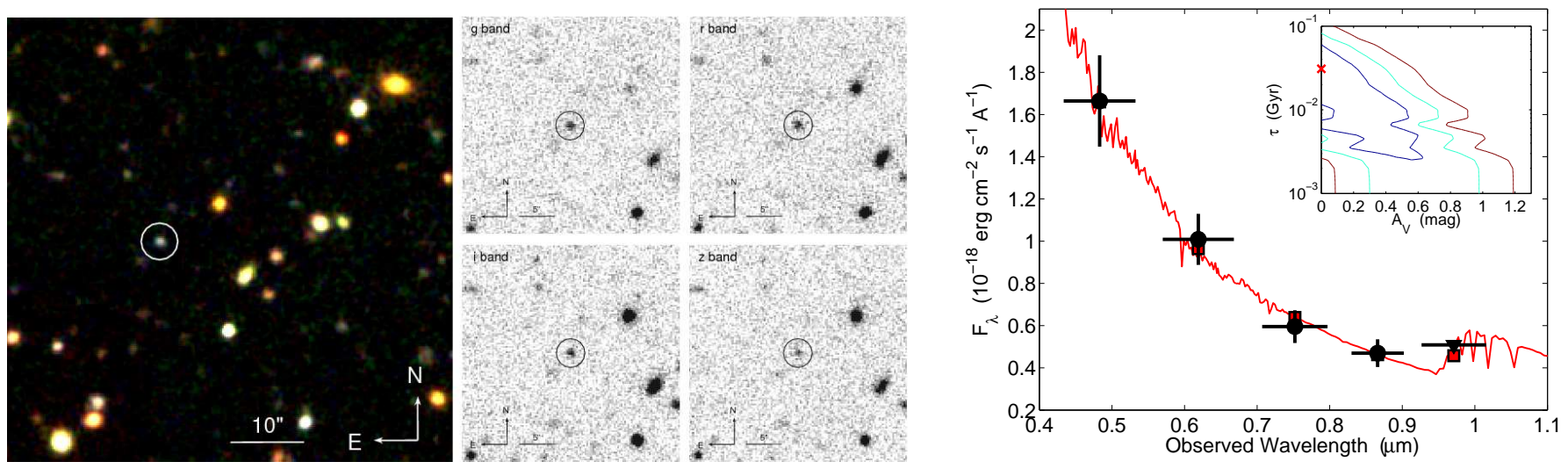

FIG. 4.- Left: PS1/MDS pre-explosion images of the host galaxy of PS1-11bam in $g_{\mathrm{P} 1} r_{\mathrm{P} 1} i_{\mathrm{P} 1} z_{\mathrm{P} 1}$, with a wider gri color-composite image demonstrating the blue colors of the host relative to nearby field galaxies. Right: Host galaxy SED (black; upper limit in $y_{\mathrm{P} 1}$ ), along with the best-fit Maraston (2005) model for $Z=0.5 \mathrm{Z}_{\odot}$, which has $\tau_{*} \approx 30 \mathrm{Myr}, M_{*} \approx 2 \times 10^{9} \mathrm{M}_{\odot}$, and $A_{V}^{\text {host }} \lesssim 0.5 \mathrm{mag}$. The inset shows the $\tau_{*}$ versus $A_{V}^{\text {host }}$ confidence regions with contours marking $1 \sigma, 2 \sigma$, and $3 \sigma$. 